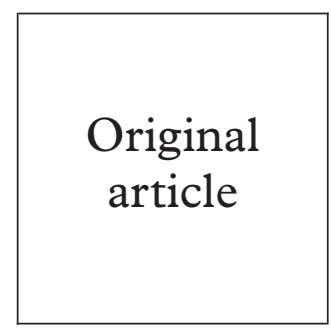

\title{
Sexually transmitted diseases and communications with general practitioners
}

\author{
Joseph Winceslaus, Jonathan Blount, Colin Cryer
}

Objectives: To devise a method of communicating with the general practitioners (GPs), overcoming the constraints imposed by patient confidentiality and the low levels of staffing in genitourinary medicine (GUM) clinics. To assess the GPs' responses to this method of communication.

Setting: GUM clinics at two centres in Kent-Maidstone and Tunbridge Wells.

Methods: Patients were recruited if they attended the clinic of their own accord without a letter of referral from their GPs; a definitive or provisional diagnosis was made and the patient was managed in the clinic; the patient's GP had received a conventional reply from the GUM clinic for other patients referred in the past. Separate GP letters were developed for male and female patients. These handwritten study letters were read by the patients who took the responsibility to deliver them to their GPs. This was followed by a questionnaire to the GPs.

Results: 75 patients were eligible. Seven patients refused to participate. All questionnaires were returned by the GPs for the 68 participating patients $(100 \%)$. Seven GPs failed to receive the study letter. For these unreferred patients, this was an improvement in communication level from $0 \%$ to $80 \%$. $79 \%$ (95\% confidence interval: $67 \%-87 \%$ ) preferred the study letter, $97 \%$ $(89 \%-99 \%)$ would like to receive a similar letter for future patients. All GPs thought that the study letter was at least as good as the standard letter, $52 \%(40 \%-64 \%)$ thought it was better. For $82 \%(70 \%-90 \%)$ it was the preferred format for future communication.

Conclusion: The study has shown a way of establishing communication with GPs for patients who do not object to this. The results also suggest that in the study districts neither the GPs nor the majority of study patients had any objection to the sharing of information between the GUM clinics and GPs.

(Sex Transm Inf 1999;75:45-48)

Keywords: STDs; general practitioners; communication

Kent and Sussex and Maidstone General Hospitals

$\mathrm{J}$ Winceslaus

Mid Kent Health Care Trust

J Blount

South East Institute of Public Health

C Cryer

Correspondence to: Dr S J Winceslaus, Department of

Genitourinary Medicine,

Kent and Sussex Hospital,

Mount Ephraim, Tunbridge

Wells, Kent, TN4 8AT.

Accepted for publication 1 October 1998

\section{Introduction}

In the United Kingdom, most patients attending genitourinary medicine (GUM) clinics, especially the large inner city ones are self referred. In Tunbridge Wells and Maidstone only $8 \%$ of new patients are directly referred by their general practitioners (GPs). GPs who have referred patients to genitourinary medicine (GUM) clinics receive a standard reply as in other specialties.

GUM clinics have now broadened their horizons and have taken on the management not only of the traditional sexually transmitted diseases (STDs), but also many other allied conditions relating to sexual health. ${ }^{12}$ Because most new STDs are of viral origin, ${ }^{3}$ often running a chronic course and are time consuming to manage, close collaboration with the GP is necessary. Additionally, to comply with the patient's charter, ${ }^{4}$ many GPs now prefer close involvement in the care of their patients including the management of traditional STDs.

The management of patients in GUM clinics is governed by the "confidentiality act". Although the act has facilitated a remarkable degree of control over STDs, it has created an atmosphere of secrecy between clinics, GPs, and their patients. This and the limited resources available to most GUM clinics ${ }^{3}$ are the main reasons why good communication links with GPs are difficult to maintain. This is especially true for the small suburban clinics many of which still remain inadequately staffed.

Literature on referral communication between GPs and hospital consultants has in the past highlighted what should be the ideal contents of GPs' letter to consultants, ${ }^{6}$ and consultants' letters to GPs, ${ }^{7-9}$ some highlighting individual problems in their specialties. ${ }^{10}$ Communication standards in GUM between consultants and GPs remain a largely neglected area. Studies on communication between GUM clinics and GPs have in the past been restricted to problems related to HIV infected patients, ${ }^{11}{ }^{12}$ but the wider issue of communication for general STDs still remains unresolved.

The purpose of the present study was to

(1) encourage better communication for general STDs with the GPs

(2) devise a method of communication that would overcome both the constraints imposed by patient confidentiality and low levels of staffing in GUM clinics

(3) assess the GP's response to this method of communication.

\section{Materials and methods}

Male and female patients attending the GUM clinics at two centres in Kent (Maidstone and Tunbridge Wells) were recruited if they met the following criteria: 
GENITO-URINARY MEDICINE

Date $03 / 09 / 95$

Dear Dr HEALER

Your patient Mr ERDS
DOB $03 / 05 / 74$
was seen in this clinic on $27 / 08 / 95$
Diagnosis: CHLAMYDIAL URETHRITIS
He complained of 1. Urethral
2. Dysured Discharge

Genital examination showed a Congested urethral meatus

and scanty wethel discharge

Gram-stained urethral smear showed evidence of moderate

Uretteitis.

Urethral test for

1. Neisseria gonorrhoeae was

Negature

2. Chlamydia trachomatis was

Positive

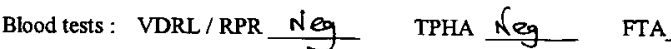
Hbsag Neg . MSU Puscellst; No growth.

Other tests/Procedures

Treatment given Doxycyaline $200 \mathrm{mg}$ daily for
ten days
Comments His regulas giel triend was treated
to-day
Yours sincerely
$\begin{aligned} & \text { Sf } \\ & \text { Dr SJ Winceslaus } \\ & \text { Consultant in Genitourinary medicine }\end{aligned}$

The Department of Genito-Urinary Medicine Preston Hallt : : oiral

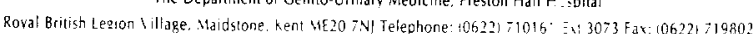

Figure 1 Letter developed for use in the study completed for a fictitious male patient. Mid Kent Healthcare Trust version.

(1) attendance at the clinic of their own accord without a letter of referral from their GPs
(2) a definitive or provisional diagnosis made and managed in the clinic

(3) their GP had received a conventional reply from the GUM clinic for other patients previously referred by them (to facilitate comparison)

(4) patient consented to participation in this study.

Separate GP letters were developed for male and female patients. These study letters contained information that is routinely presented in standard letters to GPs, but in a completely structured format. The letters were completed by hand and given to the patient for approval. Any information found unacceptable to the patient was deleted. Note was also made of any pending laboratory investigations. Two such letters for fictitious patients are shown in figures 1 and 2 .

It was made clear to the patients that the clinic would be subsequently approaching the GPs for their views about this new style of communication. This gave the patients a second chance to opt out of the study if they had any misgivings about their clinic details being sent to their GP. Patients were asked to take the letter to their GP after a copy had been filed.

After an interval of 2-3 weeks, a questionnaire and blank male and female study letters were sent to the GP accompanied by a letter explaining the purpose of the study. If no reply was received after 2 weeks, the GP was telephoned and encouraged to return the questionnaire even if the patient had failed to hand in the study letter.

\section{Results}

Seventy five patients were recruited, seven of whom refused to participate, leaving 68 patients in the study. None of them made any changes to the information contained in the study letters. Seven of the GPs did not receive the study letter from the patient. All the questionnaires were returned for the participating 68 patients by the GPs $(100 \%)$.

The results are summarised in table 1 . All 61 GPs who received the study letter thought the

Table 1 Summary of GP responses to questionnaire

\begin{tabular}{|c|c|c|c|c|c|c|}
\hline & $n$ & Yes & No & & $\%$ & $95 \% C I$ \\
\hline GP received the study letter & 68 & 61 & 7 & & 90 & \\
\hline $\begin{array}{l}\text { GP preferred the study } \\
\text { letter }\end{array}$ & $61^{\star}$ & 48 & 11 & & 79 & $67 \%-87 \%$ \\
\hline $\begin{array}{l}\text { GP would like to receive } \\
\text { similar letter for future } \\
\text { patients }\end{array}$ & 61 & 59 & 2 & & 97 & $89 \%-99 \%$ \\
\hline \multirow{2}{*}{$\begin{array}{l}\text { GP always reads entire } \\
\text { contents of the standard } \\
\text { letter }\end{array}$} & 61 & 55 & 6 & & 90 & $80 \%-95 \%$ \\
\hline & $n$ & Better & Same & Worse & $\%$ Better & $95 \% C I$ \\
\hline \multirow{2}{*}{$\begin{array}{l}\text { GP thinks that information } \\
\text { provided by the study } \\
\text { letter better than standard }\end{array}$} & 61 & 32 & 29 & 0 & 52 & $40 \%-64 \%$ \\
\hline & $n$ & Preferred study & $\begin{array}{l}\text { No } \\
\text { preference }\end{array}$ & $\begin{array}{l}\text { Preferred } \\
\text { standard }\end{array}$ & $\%$ Preferred study & $95 \% C I$ \\
\hline \multicolumn{7}{|l|}{$\begin{array}{l}\text { Future communications to } \\
\text { use the study rather than } \\
\text { standard letter }\end{array}$} \\
\hline From GUM clinics & 61 & 50 & 2 & 9 & 82 & $70 \%-90 \%$ \\
\hline From other specialties & 61 & 10 & 0 & 51 & 16 & $9 \%-28 \%$ \\
\hline
\end{tabular}

^Two GPs had no preference. 


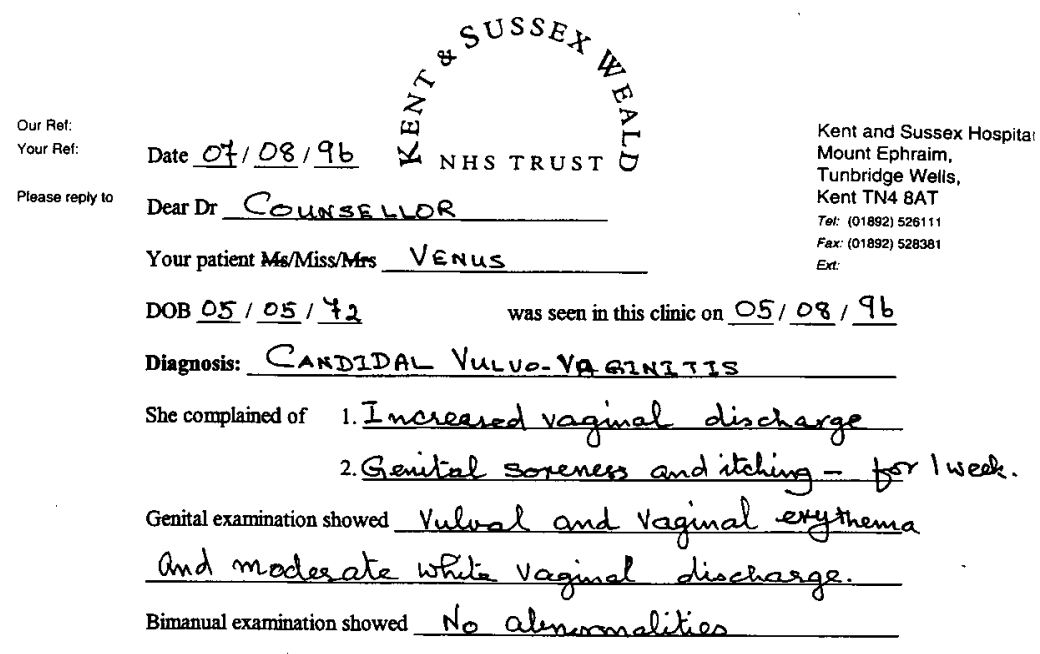

Results of Gram-stained smears. Mienoscery of Vaginal

discharge showed mycelia and spores.

Genital test for 1. Neisseria gonorrhoea $\frac{\text { Negative }}{\text { 2. Chlamydia trachomatis Negative }}$ 3. Candida isolated from HVS
4. Mixed Anaerobes Negative 5. Trichomonas vaginalis Negative

Blood tests: VDRL Negative TPHA Negative FTA

Other tests/Procedures Cerrieal Cytology-Negative (PR, 7138612)

Treatment given Comerten Vaginal persary $500 \mathrm{mg} \times 1$

Canedin cream $30 \mathrm{~g}$.

cream on the peins before resuning Sex

$\begin{array}{ll}\text { Yours sincerely } & \text { This report/document conteains } \\ \text { confidential information which is } \\ \text { subject to the veneral disease } \\ \text { regulations and further disclosure of } \\ \text { this information is restricted. }\end{array}$

Consultant in Genitourinary medicine

Figure 2 Letter developed for use in the study completed for a fictitious female patient. Kent and Sussex Weald version.

information provided was adequate (one thought that there was too much information). No additional information was thought necessary and no improvement suggested. Seventy nine per cent (95\% confidence interval: $67 \%$ $87 \%)$ preferred the study letter, $97 \%(89 \%-$ $99 \%$ ) would like to receive a similar letter for future patients, and all thought that the study letter was as good as the standard letter, with $52 \%(40 \%-64 \%)$ saying it was better. For $82 \%$ $(70 \%-90 \%)$ the study letter was the preferred format for any future communication from the GUM clinic.

GPs were asked for their comments upon the study letter, and 27 replies were received. Seven GPs felt that the letters should be posted and not hand delivered by the patient. Eight GPs provided positive comments on the study letter and particularly liked the format, clear reporting, ease of reading, and clarity. Five GPs wanted further follow up letters for patients whose treatment was continuing at the clinic. Three GPs had minor concerns about information from GUM clinics entering patient's records. Four GPs commented that the study letter should not be seen as replacing standard letters.

\section{Discussion}

In the absence of this study, for the 75 patients initially recruited and who were not referred to the clinic by their GP, there would have been no communication between the clinic and the GP. As a result of the study, however, for 61 of the 75 patients the GPs received information about the relevant conditions and management. This represents an improvement in the level of communication for unreferred patients from $0 \%$ to $80 \%$.

The other principal findings of this work are that $79 \%$ of GPs preferred the GP letter developed for this study to the standard letter, with $97 \%$ requesting similar letters for their future patients attending the GUM clinic. For information provided, all GPs thought the study letter was as good as the standard letter, with $52 \%$ showing a preference for the study letter. For $82 \%$ it was the preferred format in future communications (table 1).

The results of the present study are in conflict with the long held belief that patients do not like their GPs to know about their attendance at GUM clinics. Only seven of the original 75 eligible patients refused to take part in the study. A further seven agreed to participate but did not take the letters to their GPs. However, it cannot be assumed that the reason for this was to keep the information from their GPs as they had already consented to the clinic sending a questionnaire to the GP about the study letter.

Six per cent of GPs admitted that they did not always go through the entire contents of the standard replies sent from clinics. A letter that is quick to read and easy to assimilate would overcome these problems for the GP, provided that it contained all the important information.

Will completing these letters further impinge on clinic time of the already extremely busy GUM consultant? Most of the study letters took approximately 4-6 minutes to complete. However, as the need to communicate with GPs will be used selectively, this represents a relatively small amount of clinic time which is well spent.

In conclusion, in the two districts the study has shown a way forward in establishing effective communication links with the GPs for patients who do not object to this. This can be achieved within the current resources available to most clinics and to the satisfaction of the majority of GPs. An improvement in the level of communication with GPs, which is within the grasp of all GUM clinics, is highly desirable in view of the GPs' need for information in the new primary care led NHS.

One of the authors (CC) was supported by the NHS Executive (South Thames).

Contributions: SWJ, the principal investigator for this project, planned, initiated, and conducted all of the material research and data collection. JB participated in formulating the research letters and questionnaires, wrote database for collection of data, and helped with editing the paper. CC was responsible for the interpretation and analysis of data as well as helping with the writing and editing of the paper.

1 Sonnex C, Carne C, Edwards S. Rethinking sexual health clinics: patients prefer to have non-descriptive titles (letter). BMF 1995;310:1194-5. 
2 Stedman Y, Elstein $M$. Rethinking sexual health clinics. BMF 1995;310:342-3.

3 Monks Working Party. Report of the working group to examin workloads in genitourinary medicine clinics. London: Department of Health, November, 1988.

4 Department of Health. The patient's charter: raising the standard. London: HMSO, 1991.

5 Department of Health. National Health Service (Venereal Diseases) Regulations 1974 (SI 1974129) regulation 2. London: HMSO, 1974

6 Newton J, Eccles M, Hutchinson A. Communication between general practitioners and consultants: what should between general practitioners and consultant:

7 Rawal J, Barnett P, Lloyd B. Use of structured letters to improve communications between hospital doctors and general practitioners. BMF 1993;307:1044.

Lloyd B, Barnett P. Use of problem lists in letters between hospital doctors and general practitioners. BMF 1993;306: 247.

9 Cornwall P. Communication between general practitioners and child psychiatrists. BMf 1993;306:692-3.

10 Hodge JAH, Jacob A, Ford MJ, et al. Medical clinic referra letters. Do they say what they mean? Do they mean what they say? Scott Med f 1992;37:179-80.

11 Guthrie B, Stinton F, Barton S, et al. Communication with general practitioners by hospital based HIV units (letter). Int I STD AIDS 1994;5:458-9.

12 Guthrie B, Barton S. HIV at the hospital/general practice interface: bridging the communication divide. Int $f$ STD AIDS 1995;6:84-8. 